AGGRESSIVE BEHAVIOR

Volume 24, pages 399-409 (1998)

\title{
Attention Allocation and Habituation to Anger-Related Stimuli During a Visual Search Task
}

\author{
Dale J. Cohen, ${ }^{1 *}$ Christopher I. Eckhardt, ${ }^{2}$ and Klaus D. Schagat ${ }^{1}$ \\ ${ }^{1}$ Department of Psychology, University of North Carolina at Wilmington, Wilmington, \\ North Carolina \\ ${ }^{2}$ Department of Psychology, Southern Methodist University, Dallas, Texas
}

: : : : : : : : : : : : : : : : : : : : : : : : : : : : : : : :

\begin{abstract}
A substantial amount of data has accumulated demonstrating that emotionally disordered subjects are prone to bias their attention toward threatening, emotionally relevant stimuli. Little attention has been reserved for the study of cognitive processes involved in anger arousal. In the present study, we investigated whether mood-congruent attentional biases could be demonstrated in subjects of varying levels of trait anger using a visual search task. This task also assessed whether mood-congruent biases diminished with repeated exposure to specific emotion stimuli. To investigate state-trait interaction effects, a naturalistic, anger-inducing insult was administered to half the subjects. There was a positive relation between participants' level of trait anger and their degree of mood-congruent attentional bias toward anger-related cues only after an insult. As predicted, this effect diminished across blocks of trials. Aggr. Behav. 24:399_ 409, 1998. ๑1 1998 Wiley-Liss, Inc.
\end{abstract}

: : : : : : : : : : : : : : : : : : : : : : : : : : : : : : : : :

Key words: visual search; information processing; anger; cognition

\section{INTRODUCTION}

Numerous researchers have demonstrated that emotionally disordered subjects are prone to bias their attention toward threatening, emotionally relevant stimuli [for reviews see MacLeod and Mathews, 1991; McNally, 1995]. Although a variety of tasks have been used to investigate attentional bias, such as the dot-probe task [e.g., Broadbent and Broadbent, 1988] and word masking tasks [e.g., MacLeod and Hagan, 1992], the emotional Stroop task has been implemented with the greatest frequency. In the emotional Stroop task, subjects are asked to name the ink color of lexical stimuli of varying emotional content. Color-naming response latency is the dependent variable. In general, longer response latencies have been observed when sub-

*Correspondence to: Dale J. Cohen, Department of Psychology, University of North Carolina at Wilmington, Wilmington, NC 28403. E-mail: cohend@uncwil.edu

Received 27 May 1997; accepted 3 October 1997

() 1998 Wiley-Liss, Inc. 
jects are asked to color-name words that are related to their emotional disorder or activated emotional state, despite explicit instructions to ignore the content of the presented words. Irrespective of the task used, these mood-congruent attentional biases $^{1}$ are typically interpreted according to Bower's [1981] theory of spreading activation, which hypothesizes that the processing of emotion-relevant information activates associated memory and cognitive connections. These connections are hypothesized to initiate involuntary cognitive processes that obstruct performance. The more exaggerated the affective trait, the more affect-relevant associative connections are presumed to exist and the greater the degree of "spreading activation" when mood-congruent material is processed [Blaney, 1986].

In a recent attempt to delineate the time course of mood-congruent attentional biases, McKenna and Sharma [1995] demonstrated that greater response latencies for anxietythreat words vs. neutral-content words on the emotional Stroop task are present during early trials but diminish with repeated presentation. This habituation effect occurs at the level of the individual stimulus, i.e., after repeated presentation of specific words and not a specific class of words. These findings have important practical implications. As has been repeatedly shown in clinical research, repeatedly exposing anxious subjects to feared stimuli over a lengthy time period has the resultant effect of reducing both psychophysiological correlates of fear activation and behavioral avoidance [Foa and Kozak, 1986; Kozak et al., 1988]. The findings by McKenna and Sharma [1995] suggest that one potential mechanism through which exposure may operate is by the activation and modification of fear structures [Foa and Kozak, 1986] via repeated exposure to specific stimuli. In support of this hypothesis, Lavy and van den Hout [1993] found that spider phobics who had previously demonstrated longer color-naming latencies for spider-related stimuli relative to neutral stimuli no longer demonstrated this mood-congruent bias following a two-session exposure treatment. Thus, a critical variable to assess in the context of understanding emotional disorders and in formulating interventions for these disorders is whether a similar habituation effect can be observed by repeated exposure to emotionally valenced stimuli across numerous trials.

Although the emotional Stroop task and related paradigms have been widely applied in studies examining information processing in anxiety-disordered and depressed subjects, little attention has been reserved for the study of individuals who present with intense levels of anger. Given the recent attention afforded to anger-related public health concerns (e.g., interpersonal aggression and cardiovascular diseases), it is clear that a more systematic study of the emotion of anger is needed [DiGiuseppe, Tafrate, and Eckhardt, 1994; Tavris, 1989]. Obstructing a more focused study of individuals with anger problems is the lack of formal diagnostic categories and accepted criteria for identifying individuals with "anger disorders" [Eckhardt and Deffenbacher, 1995; Novaco, 1985]. Although several official diagnostic categories

\footnotetext{
${ }^{1}$ We note that the term mood-congruent bias "assumes that some material, by virtue of its affectively valenced content, is more likely to be stored and/or recalled when one is in a particular mood; concordance between mood at exposure and mood at recall is not required or relevant" [Blaney, 1986, p 229]. This phenomenon is not to be confused with mood dependency, which refers to an increased likelihood of remembering or processing any stimulus set (regardless of its affective tone) when there is similarity between encoding and retrieval mood state. As noted by MacLeod and Mathews [1991], the mood-congruency bias in the emotional Stroop task (and similar tasks) among emotionally disordered participants is predicted by Bower's spreading activation [1981] model since mood "should influence how readily emotional stimuli are perceived ... and will thus determine the degree to which such stimuli capture attention" [p 124].
} 
include symptoms of anger or irritability (e.g., antisocial personality disorder, borderline personality disorder, posttraumatic stress disorder), anger is not a necessary criterion. As a result, the quantity and perhaps quality of basic research on anger has been limited despite a clear need for enhanced understanding of the causes and consequences of anger arousal.

In the present study, we investigated whether mood-congruent attentional effects could be demonstrated in subjects of varying levels of trait anger using a visual search task. Although the emotional Stroop task has been one method for investigating the attentional aspects of Bower's [1981] spreading activation hypothesis, other cognitive-experimental tasks should also be able to demonstrate the attentional aspects of this model. One would expect that extraneous, task-irrelevant cognitive processes that inhibit task performance on the emotional Stroop [MacLeod, 1991] would interfere with the completion of any task requiring shared resources. In the visual search task, subjects are asked to identify the location of a neutral-content target word in a two-by-two array of lexical stimuli. The three distractor words were all anger-related, positive-emotion-related, or neutral-content words. It was predicted that high-trait anger participants' attention would be allocated toward the content of the anger-related distractor words, thus initiating task-irrelevant, extraneous cognitive processes. This would be manifested in increasing their reaction time (RT) to locate the target word in this condition. Although this task superficially differs from the standard emotional Stroop task, both tasks' susceptibility to inhibition by extraneous cognitive processes are theoretically identical.

To assess the habituation effect found by McKenna and Sharma [1995], distractor words were repeated during the experimental session. We predicted that high-trait anger subjects would exhibit longer word location latencies when the target word was interspersed with anger-emotion distractor words during early trials of the visual search task relative to their performance on neutral and positive words. We predicted no effect of distractor word type for the low-trait anger subjects. To evaluate whether moodcongruent biases in information processing are due to trait anger levels, state anger levels, or an interaction of trait and state anger, we administered a naturalistic, angerinducing insult to half the subjects. However, because researchers have reported inconsistent findings regarding mood-congruency effects when inducing a relevant mood state at the time of assessment [Broadbent and Broadbent, 1988; MacLeod and Mathews, 1988; Mathews and Sebastian, 1993; et al., 1990; Persons and Miranda, 1992], and given the absence of any such data for angry subjects, we make no prediction concerning differential RTs for insulted and noninsulted subjects.

\section{METHOD \\ Participants}

A total of 130 participants completed the experimental procedure. Participants were randomly assigned into insult or noninsult groups at the start of the experiment.

All participants were administered the Trait Anger Scale (TAS) of the State Trait Anger Expression Inventory [Spielberger, 1988] at the end of the experimental session. The State Trait Anger Expression Inventory is a well-validated measure of the experience and expression of anger that possesses excellent psychometric properties [see Fuqua et al., 1991; Spielberger, 1988; Spielberger et al., 1995]. The 10-item TAS has been used frequently to define client analog samples in treatment-outcome studies by 
Deffenbacher and colleagues [for a review see Deffenbacher, 1992] and has consistently been shown to possess excellent criterion and construct validity. Overall, no gender differences were found on the TAS (males: $\mathrm{M}=19.4, \mathrm{SD}=5.3$; females: $\mathrm{M}=18.7$, $\mathrm{SD}=4.3 ; t(128)=.772, P=.44)$. Because the TAS was given at the end of the experiment, we assessed whether the insult had an effect on the participants' TAS scores. TAS scores of the insult group $(\mathrm{M}=19.0, \mathrm{SD}=4.67)$ did not differ from those of the no insult group $(\mathrm{M}=18.7, \mathrm{SD}=4.52) ; t(126)=.33, P=.73$. The two groups (insult vs. no insult) were not significantly different with regard to age $(t(128)=-.88, P=.38 ; \mathrm{M}=$ $19.0, \mathrm{SD}=1.7)$, race $\left(\chi^{2}(4)=1.87, P=.76 ; 89 \%\right.$ white $)$, or gender $\left(\chi^{2}(1)=.039, P=\right.$ $.84 ; 73 \%$ female).

\section{Apparatus and Materials}

Stimulus words. From Clore et al.'s [1987] empirically derived taxonomy of the affective lexicon, 50 anger-related words with five to seven letters were selected (e.g., enraged, irate). The general frequency of occurrence for the 50 selected anger words was evaluated according to Thorndike and Lorge [1944]. Fifty positive-emotion words, matched for word length and frequency to the anger-emotion words, were selected from Clore et al.'s [1987] taxonomy. Two hundred matched neutral words were selected from Thorndike and Lorge [1944]. Forty nonmatched neutral words were chosen for the practice trials.

\section{Visual Search Task}

All stimuli were presented on a 15" VGA color monitor with a $60-\mathrm{Hz}$ refresh rate controlled by an 80486 microcomputer using the DOS operating system. The resolution of the monitor was $640 \times 480$ pixels.

A trial consisted of a fixation " $X$ " presented for $500 \mathrm{msec}$, followed by the target word printed in white (e.g., "chair") in the IBM default standard text. The target word was always a neutral word. The target remained on the screen for $800 \mathrm{msec}$, followed by a $16.7-\mathrm{ms}$ blank screen, followed by four words presented in a rectangular array $\left(8.5^{\circ}\right.$ visual angle horizontally, $2.5^{\circ}$ visual angle vertically. One of the four words was the previously presented target word. The remaining three distractor words were all neutral, all anger, or all positive words. The position of the target word varied randomly across trials, with the constraint of never appearing in the same location for three consecutive trials.

The subject's task was to press the key on the numeric keypad of the computer keyboard indicating the quadrant containing the target word with the dominant hand. The " 4 " key represented the upper left quadrant, the " 5 " key represented the upper right quadrant, the " 1 " key represented the lower left quadrant, and the " 2 " key represented the lower right quadrant. All other keys on the numeric keypad were removed to facilitate accurate responding. Participants' RT to identify the target word's quadrant was recorded by the computer. The next trial began immediately following the subject's response. All trials on which the subject responded incorrectly were re-presented randomly during the session using the same trial type (e.g., three neutral distractor words) but not the same words (e.g., tree, rain, and class).

A session consisted of 10 practice trials and 200 experimental trials. All distractor words in the practice trials were neutral words. For the experimental trials, 100 trials contained three anger distractor words, 50 trials contained three neutral distractor words, 
and 50 trials contained three positive distractor words. The higher percentage of anger words were used to assess any effect of habituation. The trial types were presented randomly during the session. Word selection for each trial type was random. Therefore, no subject was presented the same three-word combinations. In addition, although individual words were re-presented during the session, a given combination of three distractor words was not repeated. Target words were never repeated or used as distractors. One self-timed rest break was presented after 100 trials.

\section{Procedure}

Participants were tested in a 6' x 6' laboratory room. The experimenter explained that all tests would be completed on the computer. With the participant looking on, the experimenter completed a "login" procedure to access the program. To disguise the preinsult assessment of state anger, the following deception was used. Participants were instructed that the campus health center was conducting a study to determine the emotional effects of participating in psychology research experiments. Toward this end, participants were to complete a questionnaire from the health center that was supposedly unconnected to the experiment. This questionnaire was actually a computerized combination of the State Anger Scale [Spielberger, 1988] and State Anxiety Scale [Spielberger et al., 1970]. Items from both scales were presented in random order. To indicate their answer, participants positioned the cursor over the appropriate response and hit the "return" key. The experimenter left the room while the subject completed the scales. On completion, the experimenter returned to the room to start the visual search task. The experimenter read the instruction of the visual search task aloud and offered any additional procedural information requested by the subject. Participants were told that the testing would last 20 minutes and were instructed to contact the experimenter, who would be waiting outside the laboratory room, when the trials were completed.

To set the stage for the insult manipulation, as well as to provide an excuse for a postinsult manipulation check, a "computer malfunction" was programmed into the visual search task. After participants had completed their tenth practice trial, the computer screen was progressively and repeatedly filled with the letter " $p$ " and a high-pitched beep was repeatedly sounded. Subject were unable to terminate this apparent malfunction. All participants left the laboratory room to seek the help of the experimenter.

The experimenter entered the room and told participants to sit in front of the computer. For those participants randomly assigned to the insult condition, the researcher accused participants of doing something wrong and not following instructions. The researcher acted frustrated as he pretended to try to fix the computer problem, stating that this had never happened before and that the malfunction must have been the participants' fault. The experimenter hit a key that apparently exited the computer program to a DOS prompt, which was actually a programmed step in the "malfunction." Once the experimenter restored the initial login screen, he demanded to know why "a freshman in college can't follow directions" and whether they needed the instructions repeated yet again. Participants were told that the data were lost and that they had to "start all over." The experimenter then completed the login procedure and left the room. Participants again completed the State Anger Scale and the State Anxiety Scale, which served as a postmanipulation assessment of mood state, followed by the experimental trials.

For participants in the noninsult condition, the experimenter responded to the "computer malfunction" in a conciliatory, embarrassed manner. The experimenter pretended 
to fix the computer problem while apologizing for the "glitch." The participants were repeatedly told that the problem was erratic and had nothing to do with their performance. The apologetic experimenter asked participants whether they would mind starting over because "their data must have been lost." The experimenter then completed the login procedure and left the room. Participants again completed the State Anger Scale and the State Anxiety Scale, followed by the experimental trials.

The experimenter spent approximately 3 minutes with each subject in the laboratory room in both insult conditions following the computer malfunction. The experimenter ignored all verbal responses by participants in all conditions. Following the experimental trials, all participants were administered the TAS [Spielberger, 1988] and a demographics questionnaire. The TAS was administered following the visual search task to reduce potential priming by the scale. All participants were then debriefed. The debriefing entailed complete disclosure of the purpose of the experiment, a description of why deception was necessary, and an opportunity for participants to discuss any emotional reactions experienced during the insult or at any other point during the experimental situation. The experiment was terminated prematurely for two participants who were inappropriately distressed following the insult. These participants were debriefed and their data were disregarded. Because all participants were not given the TAS until the end of the experimental session, it was not possible to determine the trait anger level of these two participants.

\section{RESULTS}

\section{Manipulation Check}

Two- (insult vs. noninsult) by-two (premanipulation vs. postmanipulation) analyses of variance (ANOVAs) were computed using State Anger Scale scores and State Anxiety Scale scores as dependent variables (see Table I).

State Anger Scale. There was a main effect of insult, F $(1,127)=4.00, P=.04$, such that participants in the insulted condition scored higher than participants who were not insulted. There was a main effect of manipulation, $\mathrm{F}(1,127)=12.06, P<.001$, such that participants scored higher after manipulation than before manipulation. Finally, there was an insult by manipulation interaction, $\mathrm{F}(1,127)=26.78, P<.001$, such that postmanipulation insulted participants scored higher than postmanipulation noninsulted participants and all premanipulation participants. This pattern of data indicated that the insult increased participants' anger levels.

State Anxiety Scale. There was no main effect of insult, F $(1,127)=1.97$, ns. There was a main effect of manipulation, $\mathrm{F}(1,127)=6.98, P=.01$, such that participants

TABLE I. Means (SDs) on the StateAnger Scale and the StateAnxiety Scale in the Insult and Noninsult Condition Before and After the Manipulation*

\begin{tabular}{llllll}
\hline & \multicolumn{2}{c}{ State Anger Scale } & & \multicolumn{2}{c}{ State Anxiety Scale } \\
\cline { 2 - 3 } & Before insult & After insult & & Before insult & After insult \\
\hline Insulted & $12.3(3.56)^{\mathrm{a}}$ & $15.0(5.67)^{\mathrm{b}}$ & & $40.3(9.62)^{\mathrm{a}}$ & $43.0(10.31)^{\mathrm{b}}$ \\
Noninsulted & $12.5(3.82)^{\mathrm{a}}$ & $12.0(4.23)^{\mathrm{a}}$ & & $39.3(9.37)^{\mathrm{a}}$ & $39.4(10.10)^{\mathrm{a}}$ \\
\hline
\end{tabular}

*Post hoc Tukey HSD tests were calculated to determine group differences.

${ }^{a, b}$ Within scale, those groups sharing the same letter did not differ significantly $(P<.05)$. 
scored higher after manipulation than before manipulation. Finally, there was an insult by manipulation interaction, $\mathrm{F}(1,127)=5.48, P=.02$, such that postmanipulation insulted participants scored higher than postmanipulation noninsulted participants and all premanipulation participants. This pattern of data indicated that the insult also increased participants' anxiety levels.

\section{Group Differences}

To assess the effect of habituation, the data were split into two blocks of 100 trials each. ${ }^{2}$ To test the relation between trait anger and attention toward irrelevant anger stimuli, three hierarchical regression analyses were performed on participants' RTs (all RTs reported in msec) for each block. Each model tested a different hypothesis. In Model 1, we tested whether participants took longer to identify the target when surrounded by anger-related words relative to positive words by using the following effect coding of distractor words: anger words $=1$, positive words $=-1$, and neutral words $=0$. In Model 2, we tested whether participants took longer to identify the target when surrounded by anger-related words relative to neutral words by using the following effect coding of distractor words: anger words $=1$, positive words $=0$, and neutral words $=-1$. In Model 3, we tested whether participants took longer to identify the target when surrounded by positive words relative to neutral words by using the following effect coding of distractor words: anger words $=0$, positive words $=1$, and neutral words $=-1$. In all models we included participants' TAS scores, effect codes for insult (insult $=1$, noinsult $=-1$ ), and two- and three-way interactions as predictors.

Models 1 and 2 tested the hypothesis that trait anger is related to the difference in RTs between anger distractor words and positive words (Model 1) and anger distractor words and neutral words (Model 2). Model 3 assessed whether the results of Models 1 and 2 were due to the specific emotion associated with the distractor words (i.e., anger) or due to the presence of emotion content in the distractor words regardless of the specific emotion. For each effect, we report the unstandardized regression coefficient (B), standardized coefficient $(\beta)$, and the semipartial correlation $\left(s r^{2}\right)$.

Because the RT data were positively skewed (as is common to RT data), RT was transformed into inverse speed scores $(-1000 / \mathrm{RT})$, where greater scores indicate slower RTs. Since RT data are only valid if the subject does the task correctly, only correct responses were included in the analysis [Weltford, 1980]. The average subject's error rate was $1 \%$, which is quite low.

Effects common to all models. The effects that are common to all models are the main effects and interactions that do not include the distractor word variable. Therefore, time of response for the following effects includes RTs for all trials (i.e., collapsed over distractor words). In block 1 , there was a negative relation between TAS score and time of response, $\mathrm{B}=-0.005, \beta=-0.07, s r^{2}=0.063, P<.01$, such that the higher one's TAS score the faster one responds across all trials. There was also a positive effect of insult, $\mathrm{B}=0.04, \beta=0.11, s r^{2}=0.011, P<.01$, such that participants in the insult group responded slower to all trials than participants in the noninsult group. There were no other significant effects common to all models in block 1 .

${ }^{2}$ The results do not change when the trials are split into smaller blocks. Therefore, for ease of understanding we report the two-block analysis. 
In block 2, there was a negative relation between TAS score and time of response, $\mathrm{B}$ $=-0.002, \beta=-0.27, s r^{2}=0.03, P<.01$, and a positive effect of insult, $\mathrm{B}=0.013, \beta=$ $0.034, s r^{2}=0.03, P<.01$. These effects were similar to those of block 1 . There were no other significant effects common to all models in block 2 .

Model-specific effects. Model-specific effects are main effects and interactions that include the distractor word variable. Therefore, time of response is the relation between the two trial types paired in the models discussed earlier (e.g., Model 1: RT to trials involving anger distractor words in relation to positive distractor words).

Models 1 and 2. Anger words vs. positive and neutral. The results from Models 1 and 2 were essentially identical and therefore will be discussed together. There was a negative interaction between insult and distractor word, $\mathrm{B}=-0.004, \beta=-0.1, s r^{2}=0.0023$, $P<.01$, such that participants who were insulted responded faster to anger words and participants in the noninsult condition responded faster to positive and neutral words. As predicted, there was a positive three-way interaction between distractor word, insult, and TAS, $\mathrm{B}=0.002, \beta=0.11, s r^{2}=0.026, P<.01$, such that in the insult condition, as TAS scores increase, the participants' RTs to anger words increase more than their RTs to positive words. In the noninsult condition, as TAS scores increase, the participants' RTs to positive words increase more than their times to anger words. There were no other significant model-specific effects. In block 2 , as predicted, there were no interaction effects or any other significant model-specific effects. The pattern of data indicates that participants' TAS scores are positively related to their response time difference between trials with anger distractor words and trials with positive or neutral words only for block 1.

Model 3. Positive words vs. neutral. As predicted, for blocks 1 and 2 their were no significant model-specific effects indicating that the results of Models 1 and 2 were due to the specific content of the emotion word distractor (i.e., anger).

\section{DISCUSSION}

In the first block of trials of the insult condition, there was a positive relation between participants' level of trait anger and the difference in RTs to trials when distractor words were anger-related relative to neutral or positive-emotion words. No such effect was found in the second block of trials. This result suggests that high-trait anger participants allocate more attentional resources to anger-related stimuli than neutral or positive stimuli when insulted. In addition, this tendency toward mood-congruent attentional biases dissipates with increased exposure to specific anger-related stimuli.

As predicted, those participants who were insulted and higher in trait anger had longer response latencies when the target word was surrounded by anger-related distracters relative to positive-emotion distracters. Among the participants in the present study, dispositional tendencies may have interacted with the cognitive, affective, and physiological changes that accompanied the anger-inducing insult to result in the tendency to be distracted by task-irrelevant, anger-related stimuli. Thus, in an interpersonal conflict such as the insult manipulation used in the present study, high-trait anger participants may be prone to interpret the situation as possessing a high degree of hostile threat and respond with a high level of state anger. As generally predicted by the spreading activation theory of Bower [1981] and a recent update by Persons and Miranda [1992] (the "mood-state hypothesis"), the induction of anger may be necessary when investigating 
anger-related differences in cognitive processing. Individuals high in trait anger, when insulted, are more likely to selectively attend to anger stimuli because of complex and diffuse interconnections between anger activation and information processing operations. Importantly, when high-trait anger participants are not angered, these interconnections are hypothesized to remain latent.

The present study's findings regarding trait anger represent an extension of moodcongruent attentional effects previously demonstrated with anxiety- and depressivedisordered participants. Although some researchers have found that trait levels of anxiety interact with increases in state anxiety to produce mood-congruency effects [e.g., Broadbent and Broadbent, 1988], others have found the effect only in high-trait anxiety subjects exposed to a prolonged (rather than laboratory-induced) stressor [Mogg et al., 1994] and in both high- and low-trait anxiety subjects exposed to a high-stress condition [Mogg et al., 1990]. Findings from our lab have also indicated that during an emotional Stroop task, high-trait anger subjects who were angered prior to the task were more likely to show longer color-naming latencies for anger stimuli than were low-trait anger participants [Eckhardt and Cohen, in press]. Thus, although it seems that trait levels of emotion can interact with state manipulations of a relevant mood to produce the attentional biases predicted by the spreading activation model, additional research is needed to assess whether the effect is seen across emotions and cognitive assessment methodologies.

McNally [1995] suggests that the attentional biases predicted by Bower's [1981] spreading activation model represent deficits resembling (but not equivalent to) automatic, preattentive, and involuntary processing distortions. In the arena of anger research, numerous investigators have indicated that individuals high in trait anger demonstrate excesses or disturbances on measures of strategic, controlled cognitive processes, such as on questionnaire measures of Ellis' [1962] irrational beliefs [Ford, 1991; Hazaleus and Deffenbacher, 1985; Hogg and Deffenbacher, 1986; Kassinove and Eckhardt, 1994; Lohr et al., 1988; Mizes et al., 1990] or when asked to provide causal attributions of ambiguous interpersonal situations [Holtzworth-Munroe and Hutchinson, 1993; Slaby and Guerra, 1988]. The present investigation provides preliminary evidence of biases in preattentive information processing during anger arousal among individuals with high levels of trait anger.

The tendency of participants high in trait anger to allocate attention toward emotional stimuli decreased with repeated exposure to those stimuli. This effect is similar to McKenna and Sharma's [1995] findings that nonclinical participants' tendency to take longer to color-name threat words diminished as they were repeatedly exposed to the same threat words across blocks of trials. The participants assessed in the present study showed the same pattern of results. Several authors [Lavy and van den Hout, 1993; McNally, 1995] have noted that the effectiveness of exposure-based interventions for anxiety-disordered patients may be due to habituation of their involuntary, automatic tendency to allocate attentional resources in the direction of threatening stimuli. The present data, although preliminary and in need of replication, suggest that individuals whose primary clinical problem involves intense anger arousal may also benefit from exposure-based interventions that target these involuntary cognitive processes. Several authors have commented that given its theoretical tenability, an exposure-based treatment for angry patients represents a promising, but as yet unfulfilled, treatment alternative [DiGiuseppe et al., 1994; Tafrate, 1995]. 
In conclusion, the present study provides suggestive evidence that angry participants involuntarily allocate attention toward anger-related cues. Furthermore, this effect diminishes with repeated exposure to specific anger-related stimuli. It would be interesting to investigate the behavioral correlates of such biases. For example, MacLeod and Hagan [1992] examined anxiety-related mood-congruent attentional effects among women undergoing tests for cervical pathology. Of those women subsequently testing positive for the pathology, mood-congruent response inhibition on the emotional Stroop task were found to be the stronger predictor of negative emotional reactions 6 months after the diagnosis compared with questionnaire measures of anxiety and depression. One could hypothesize that those individuals demonstrating anger-related mood-congruent biases are more likely to interpret situations in a hostile, threatening manner and exhibit higher levels of trait anger and aggressive behavior. In addition, researchers can also address whether both the mood-congruent response inhibition and anger-related behavioral dysfunction diminish following interventions that repeatedly expose participants to anger-provoking stimuli.

\section{ACKNOWLEDGMENTS}

Portions of this manuscript are based on Klaus D. Schagat's honor's thesis submitted to the Department of Psychology, University of North Carolina at Wilmington, under the direction of Christopher I. Eckhardt.

\section{REFERENCES}

Blaney P (1986): Affect and memory: A review. Psychological Bulletin 99:229-246.

Bower GH (1981): Mood and memory. American Psychologist 36:129-148.

Broadbent DE, Broadbent MHP (1988): Anxiety and attentional bias: State and trait. Cognition and Emotion 2:165-183.

Clore GL, Ortony A, Foss MA (1987): The psychological foundations of the affective lexicon. Journal of Personality and Social Psychology 53:751-766.

Deffenbacher JL (1992): Trait anger: Theory, findings, and implications. In Butcher JN, Spielberger CD (eds): "Advances in Personality Assessment." Vol. 9. Hillsdale, NJ: Lawrence Erlbaum, pp 177-201.

DiGiuseppe R, Tafrate R, Eckhardt CI (1994): Critical issues in the treatment of anger. Cognitive and Behavioral Practice 1:111-132.

Eckhardt CI, Cohen DJ (1997): Attention to angerrelevant and irrelevant stimuli following naturalistic insult. Personality and Individual Differences 23:619-623.

Eckhardt CI, Deffenbacher J (1995): Assessment of anger disorders. In Kassinove H (ed): "Anger Disorders: Definition, Diagnosis, and Treatment." Washington, DC: Taylor \& Francis, pp 27-48.
Ellis A (1962): "Reason and Emotion in Psychotherapy." New York: Lyle Stuart.

Foa EB, Kozak MJ (1986): Emotional processing of fear: Exposure to corrective information. Psychological Bulletin 99:20-35.

Ford BD (1991): Anger and irrational beliefs in violent inmates. Personality and Individual Differences 12:211-215.

Fuqua DR, Leonard E, Masters MA, Smith RJ, Campbell JL, Fischer PC (1991): A structural analysis of the State-Trait Anger Expression Inventory. Educational and Psychological Measurement 51:439-446.

Hazaleus SL, Deffenbacher JL (1985): Irrational beliefs and anger arousal. Journal of College Student Personnel 26:47-52.

Hogg JA, Deffenbacher JL (1986): Irrational beliefs, depression, and anger among college students. Journal of College Student Personnel 27:349-353.

Holtzworth-Munroe A, Hutchinson G (1993): Attributing negative intent to wife behavior: The attributions of maritally violent versus nonviolent men. Journal of Abnormal Psychology 102:206-211.

Kassinove H, Eckhardt CI (1994): Irrational beliefs and self-reported affect in Russia and America. Personality and Individual Differences 16:133-142. 
Kozak MJ, Foa EB, Steketee G (1988): Process and outcome of exposure treatment with obsessive-compulsives: Psychophysiological indicators of emotional processing. Behavior Therapy 20:157-169.

Lavy E, van den Hout M (1993): Selective attention evidenced by pictorial and linguistic Stroop tasks. Behavior Therapy 24:645-657.

Lohr JM, Hamberger LK, Bonge D (1988): The nature of irrational beliefs in different personality clusters of spouse abusers. Journal of Rational-Emotive and Cognitive Behavioral Therapy 6:273-285.

MacLeod CM (1991): Half a century of research on the Stroop effect: An integrative review. Psychological Bulletin 109:163-203.

MacLeod CM, Hagan R (1992): Individual differences in the selective processing of threatening information, and emotional responses to a stressful life event. Behavior Research and Therapy 30:151-161.

MacLeod CM, Mathews AM (1988): Anxiety and the allocation of attention to threat. Quarterly Journal of Experimental Psychology: Human Experimental Psychology 38:659-670.

MacLeod CM, Mathews AM (1991): Cognitiveexperimental approaches to the emotional disorders. In Martin PR (ed): "Handbook of Behavior Therapy and Psychological Science: An Integrative Approach." Elmsford, NY: Pergamon, pp 116-150.

Mathews AM, Sebastian S (1993): Suppression of emotional Stroop effects by fear-arousal. Cognition and Emotion 7:517-530.

McKenna FP, Sharma D (1995): Intrusive cognitions: An investigation of the emotional Stroop task. Journal of Experimental Psychology: Learning, Memory, and Cognition 21:1595-1607.

McNally RJ (1995): Automaticity and the anxiety disorders. Behavior Research and Therapy 33:747-754.

Mizes JS, Morgan GD, Buder J (1990): The relationship between cognitions, assertion, and anger arousal. Journal of Cognitive Psychotherapy: An International Quarterly 4:369-376.
Mogg K, Bradley BP, Hallowell N (1994): Attentional bias to threat: Roles of trait anxiety, stressful events, and awareness. The Quarterly Journal of Experimental Psychology 47:841-864.

Mogg K, Mathews A, Bird C, Macgregor-Morris R (1990): Effects of stress and anxiety on the processing of threat stimuli. Journal of Personality and Social Psychology 59:1230-1237.

Novaco RW (1985): Anger and its therapeutic regulation. In Chesney MA, Rosenman RH (eds): "Anger and Hostility in Cardiovascular and Behavioral Disorders." Washington, DC: Hemisphere, pp 203-226.

Persons JB, Miranda J (1992): Cognitive theories of vulnerability to depression: Reconciling negative evidence. Cognitive Therapy and Research 16:485-502.

Slaby RG, Guerra NG (1988): Cognitive mediators of aggression in adolescent offenders: 1. Assessment. Developmental Psychology 24:580-588.

Spielberger CD (1988): "Manual for the State Trait Anger Expression Inventory." Odessa, FL: PAR.

Spielberger CD, Gorsuch RL, Lushene RE (1970): "Manual for the State Trait Anxiety Inventory." Palo Alto, CA: Consulting Psychologists Press.

Spielberger CD, Reheiser EC, Sydeman SJ (1995): Measuring the experience, expression, and control of anger. In Kassinove $\mathrm{H}$ (ed): "Anger Disorders: Definition, Diagnosis, and Treatment." Washington, DC: Taylor \& Francis, pp 49-67.

Tafrate R (1995): Treatment strategies for adult anger disorders: A quantitative review of the literature. In Kassinove H (ed): “Anger Disorders: Definition, Diagnosis, and Treatment." Washington, DC: Taylor \& Francis, pp 109-130.

Tavris C (1989): "Anger: The Misunderstood Emotion." New York: Touchstone.

Thorndike EL, Lorge I (1944): “The Teacher's Word Book of 30,000 Words." New York: Bureau of Publications.

Weltford AT (1980): "Reaction Times.” New York: Academic Press. 